\title{
The backbone of our chemical computations
}

\author{
Terry Richard Stouch ${ }^{1}$ D
}

Received: 6 February 2019 / Accepted: 14 February 2019 / Published online: 22 February 2019

(c) Springer Nature Switzerland AG 2019

It is JCAMD's particular pleasure and honor to present the two leading papers in this issue. They address the foundation of much of our computational chemistry computations: the mathematics and physics underlying our computational engines and the inner cogs and levers that give us chemical structure, energy, and dynamics. The subject is at the core of much of our work and if it doesn't work-and work wellour results can be meaningless or misleading.

Of course, I'm talking about force fields.

I'm fortunate to have observed computational chemistry in the early days when presentations - although with an eventual goal to provide some unknown knowledge of chemistry and biology - for the most part concentrated on the raw physics of atoms and molecules, experimental variables, molecular energetics, and even, at the time, the difficult task to employ this knowledge to develop force fields. It was an inspiration to hear brilliant colleagues finding a way to master physics and chemistry into useful force fields despite their relative simplicity.

Unfortunately, more and more force fields have become shrouded by the software that we use and often they are given little, if any, thought by the users. It's disturbing to see that some computational chemistry users mix portions of force fields with the presumed goal of matching pieces of different force fields in order to get a calculation running when available force fields fall short.

An extremely bright colleague once claimed that force field development is straightforward that it is "simply" a mathematical fitting process. Painfully, he found out differently. The development of force fields of physical value has not been easy. Force fields are involved and complex. And it's seldom that we know the errors of their resultant calculations or even those of some potentially important details,

Terry Richard Stouch—Senior Editor-in-Chief Journal of Computer-Aided Molecular Design.

Terry Richard Stouch

tstouch@gmail.com

1 Science For Solutions, LLC, West Windsor, NJ, USA such as the energy barrier of individual torsion angles. Further, let's assume that the error of each force field component of a molecule might be only a fraction of $\mathrm{kcal} / \mathrm{mol}$ but that the molecule as a whole contains dozens of such components. What is the overall error? Just how accurate are our results?

We are very fortunate that our current force fields work as well as they appear to do. But do they give us the accuracy that we need? How would we know? Isn't it time for us to consider this?

On that somewhat dismal note, I encourage everyone who uses force fields to read the two leading papers of this issue. For some these will be an excellent foundation to understanding force fields and their development and use. For those more experienced in the area the papers will serve as a great refresher. Although one might not agree with all the conclusions drawn in the work, anyone involved in either of these pursuits will benefit from the work covered in these papers.

Several knowledgeable colleagues were kind enough to spare the time to review these papers in depth, note some of the comments:

Rev 1:

This is a fascinating manuscript, well written, insightful, carefully referenced, and intellectually balanced. It seems that all technical terms are carefully introduced. This should be required reading for anyone who starts to develop or modify a force field. It will also be interesting reading for anyone who uses force fields. I am looking forward to the next part of this survey.

Rev 2:

This manuscript gives a magnificent review of biomolecular force field development from its inception up through the early new millennium. The review is accompanied by incisive and insightful analysis of the individual force fields and meta-analysis of important larger factors, such as the importance of the inclusion of diverse sources of data in fitting and validation, and 
how the omission of coupling terms (at least some key ones) introduces profound limitations on the accuracy and generality of the final product. This review will be essential reading for anyone starting out in the area of biomolecular force field development and very helpful background for those who use them. It was a long but delightful read, and I see value for the community in retaining it all.

\section{Rev 3:}

This is an important review, if the next generation of FF developers are to avoid (p35) the vicious cycle which has led to the proliferation of FFs over the last decades and be able to benefit from the accumulated wisdom of earlier FF derivations.

It will [also] be a valuable reference for those using FFs.

The observation that FF development is like "whack a mole" as improving the prediction of one property leads to the deterioration of others is well illustrated by this account. Too many assumptions are just not accurate enough for modelling peptide behavior.

There is great coverage of the types of force-field that are meant for modelling biomolecules and their interactions ...

I said that it was an honor to present these papers. Indeed I cannot be anything but impressed by the amount of time, consideration, contribution, and knowledge that is shared with us by the author of these leading papers, Arnold T. Hagler (Arnie) who has been working on these particular papers for several years and who has studied the content for about five decades.

Arnie is well known in the force field community and has been working on force fields since the 1970s. He was a student who stood on the shoulders of even earlier (and if I may say, revered) pioneers of the field: Schneir Lifson, of the Weismann Institute, and Harold Scheraga, of Cornell University. Their work is also well worth reading. Arnie has published over 147 papers many on the development of force fields, while others focus on their application to biological systems and structure based drug discovery.

Arnie has been and continues to be very active and involved. Among his many accomplishments, he has held a number of positions including faculty and several other positions at the Weizmann Institute and visiting Scholar or Adjunct Professor at a number of universities including several of the University of California sites, the University of Massachusetts, Amherst, and the Shanghai Institute of Organic Chemistry. He's been a consultant, a member of the Board of Directors, and/or a member of the Advisory Board of more than six companies and organizations. As an entrepreneur he has been the founder of Science Media, the
Corporate VP and President of Structure Based Design at Discovery Partners, and the Chairman of Biophysics of the Agouron Institute.

However, Arnie perhaps is best known, at least to me, as the founder of Biosym Technologies, Inc. along with Donald Mackay. Biosym was one of the first computational chemistry software companies. Biosym grew rapidly and to a size of 240 employees. They developed and managed many substantial biophysics and drug discovery software tools. Over time Biosym has evolved through several companies and is currently known as BIOVIA.

An important part of Biosym was the pioneering implementation of one of the first, if not the first, consortiums within a computational chemistry software company for development of the next generation of force fields. It was dedicated to the development of the CFF (Consistent Force Field) that grew at least in part from the CVFF (Consistent Valence Force Field) that had been developed earlier by Arnie, Schneir Lifson, and others (including Mordechai Bixon and Nobel Laureate Arieh Warshel).

The Consortium (PEFC (Potential Energy Force field Consortium)), grew rapidly in terms of participation and membership and reached 30 members including pharmaceutical and materials and hardware companies as well as nonprofit organizations and academics. Most of these contributed funds as well as time, effort, experience, computer time, and data. With such support, Arnie was able to do something else that he does well, he assembled within Biosym a substantial group of exceptional colleagues who contributed experience and drive and made the substantial contributions that made the effort work.

Perhaps more so than many force field developments of the time, the PEFC had clear goals, plans, and strategy. The ultimate aim, which was largely completed over several years (work began in the late 1980s and progressed to the late 1990s), was to develop a force field that would cover important functional moieties pertinent to pharmaceutical research as determined by the Consortium partners. This was not an easy task. Much work had been done with protein force fields in several laboratories, but the range of functionality of proteins is paltry compared to that of compounds of interest to drug development.

A systematic approach was developed early. Software was developed to partially automate the process. (Merck was a member of the PEFC during the software development and then used the software for development of the well-known and publicly distributed MMFF force field). The form of the force field was well-determined early. Data for the fitting of individual functional groups was largely determined by, at those times, very significant quantum mechanics $(\mathrm{QM})$ calculations.

Results of the force field results were validated via comparison of the results to the QM data and to small molecule 
crystal structures. Validation was done by statistical comparison of the force field with experiment. Consequently, users were aware of the reliability of the result. How many force fields that you use come with error estimates? How many force fields are identified as to their proper use?

Optimization was carried out by the novel method of fitting QM energies, forces and Hessians. The "force balance" method is a special case of this procedure (with zero weights for hessian and energy), which basically anticipated force balance by $\sim 20$ years.

Compared to today, the available amount of computer power was limited and slow and data storage was slim. Consequently, there were some that said the Consortium's goals could not succeed; it was said that the goals were too grand, the computing power was too limited, and the storage capability was too small. They were wrong. They did not credit the PEFC for its substantial consideration, planning, strategy, and determination.

The Consortium was a substantial success. The force field covered all of the functional groups that I used for many years in drug design efforts, far beyond other force fields of the time, and were always well-validated by comparison to crystal structures and quantum mechanics results. The unfortunate aspect of the effort was that, at least at the time, was not made public and also that the form of the force field, as you will see, has additional complexity that requires more coding in exchange for better results.

Arnie's two papers are, in fact, not about Biosym, and although CFF and to a lesser extent CVFF are discussed, the two papers go far beyond them. The papers are about force fields in general (about 10 active force fields from many laboratories are discussed), and about physics, chemistry, accomplishments, and history. They cover the physical basis behind such approximations as the experimental data that led to the use of a negligible radius on polar hydrogens, and the physics underlying FFs, which need to be accounted for if we are to have accurate, reliable results including forces resulting from charge flux, polarizability and anisotropy of nonbond interactions among others.

But enough commentary. Take a look at the reviewers' comments and then dive into Arnie's articles. Although the articles are long and involved they are interesting, enlightening, and informative; it is well worth the time to read them. Arnie has provided us with an exceptional consolidation of years of information all in one place. He's included history, deep knowledge, opinion and a very large amount of his time and effort, and, as you will see, even a round of whack-a-mole!

Publisher's Note Springer Nature remains neutral with regard to jurisdictional claims in published maps and institutional affiliations. 